\title{
HIGH-VELOCITY CLOUDS AND GALACTIC EVOLUTION
}

\author{
H. VAN WOERDEN, U.J. SCHWARZ, R.F. PELETIER \\ Kapteyn Institute, University of Groningen \\ Postbus 800, 9700 AV Groningen, The Netherlands \\ AND
}

B.P. WAKKER

Dept. of Astronomy, Univ. of Wisconsin, Madison WI, USA

High-velocity clouds (HVCs) are HI clouds deviating strongly from the general Galactic rotation. Their distances, and hence origins, have long remained unknown. However, three major complexes are now known to lie at $\mathrm{z}>1 \mathrm{kpc}$, while two smaller clouds are closer to the plane (Wakker et al. 1995). These incomplete results still allow a variety of origins.

$\mathrm{Ca}^{+}$and other metal ions have been found in at least seven HVCs (see reviews by Wakker et al. 1995; Schwarz et al. 1995). Abundances vary from cloud to cloud and from ion to ion, with values relative to solar ranging from 0.002 to about 1 . These variations may be due to different origins, ionization conditions, and depletion of metals onto dust. Clearly, these HVCs are not primordial; they may consist of enriched intergalactic gas, or depleted Disk gas circulated into the Halo, or a mixture of both.

We have detected CaII absorption by the largest HVC,Complex $\mathrm{C}$, in the quasars PG1351+640 and Mark 290 (Wakker et al. 1996), giving $\mathrm{Ca}^{+} / \mathrm{HI}$ ratios of 0.007 and 0.012 times the solar total calcium abundance, similar to those found in the Magellanic Stream and in Complex A.

The detection of $\mathrm{Ca}^{+}$in Complex $\mathrm{C}$ opens the road to a determination of its distance and nature. Further work will clarify the origin of HVCs, and their role in the evolution of the Galaxy and its interstellar medium.

\section{References}

Schwarz, U.J., Wakker, B.P. van Woerden, H. 1995, A\&A 302, 364

Wakker, B.P., van Woerden, H., Schwarz, U.J., Peletier, R.F., Douglas, N.G., Danly, L., de Boer, K.S. 1995, IAU Symp. 169, in press.

Wakker, B.P., van Woerden, H., Schwarz, U.J., Peletier, R.F., Douglas, N.G. 1996, A\&A (Letters), submitted. 